\title{
Langevin Dynamics of a Polymer with Internal Distance Constraints
}

\author{
Michael P. Solf, Thomas A. Vilgis \\ Max-Planck-Institut für Polymerforschung, Postfach 3148, 55021 Mainz, Germany
}

(March 31, 2018)

\begin{abstract}
We present a novel and rigorous approach to the Langevin dynamics of ideal polymer chains subject to internal distance constraints. The permanent constraints are modelled by harmonic potentials in the limit when the strength of the potential approaches infinity (hard crosslinks). The crosslinks are assumed to exist between arbitrary pairs of monomers. Formally exact expressions for the resolvent and spectral density matrix of the system are derived. To illustrate the method we study the diffusional behavior of monomers in the vicinity of a single crosslink within the framework of the Rouse model. The same problem has been studied previously by Warner (J. Phys. C: Solid State Phys. 14, 4985, (1981)) on the basis of Lagrangian multipliers. Here we derive the full, hence exact, solution to the problem.

61.41.+e, 64.60.Cn, 87.15.By
\end{abstract}

Typeset using REVTEX 


\section{INTRODUCTION}

A theoretical treatment of the dynamics of polymer networks is a generally unsolved problem. In a preliminary attempt Edwards et al. [1,2] studied the problem of a polymer subject to internal distance constraints. In their investigation the underlying theoretical problem was to handle the quenched degrees of freedom (hard crosslink constraints) which, for example, in a random network exist between pairs of arbitrary polymer segments (the monomers). As a first step Edwards considered a (macroscopically) long polymer chain which was internally crosslinked to itself at random. The polymer backbone was assumed to be Gaussian and the resulting dynamics was found to be of the standard Rouseian type [3, 4]. Permanent junction points were treated by Lagrangian multipliers, which led to enormous technical difficulties for the corresponding differential equations. In fact, these could only be handled by strong approximations, such as pre-averaging in combination with harmonic variations. Even when the problem was highly oversimplified and only one crosslink was considered the method of Lagrangian multipliers still becomes highly involved as was pointed out in a successive paper by Warner [5].

The purpose of the present paper is to develop an alternative formalism for treating Langevin dynamics of polymers subject to internal distance constraints. For calculational simplicity the simplest working model for a free polymer, the Rouse model [3, 4 , is considered. It is suggested that the more complicated problem of a random network can also be treated exactly by the presented method. We adopt here the minimal model suggested by Edwards [1,2] and consider one (macroscopically) huge polymer chain which is randomly crosslinked to itself. Such a crosslinking process will lead to tetrafunctional crosslinks. In previous works we have already demonstrated that the analogous static problem can be solved exactly when excluded volume effects between the polymer segments are ignored [6,77]. Physical quantities such as the static structure factor or the radius of gyration were found to be self averaging and could be determined by relatively simple numerical means. The essential trick was to account for the crosslinks in a general connectivity matrix, that includes both the connectedness of 
the polymer chain and an additional contribution from the crosslinking. In close analogy we expect the corresponding dynamic problem to have a similar exact solution as long as complicating factors such as excluded volume, hydrodynamic forces or entanglements are neglected. To demonstrate this analogy we start from the standard Langevin description for the polymer segments and solve the stochastic differential equation in terms of its resolvent. As an instructive example we reconsider the eight-shaped polymer problem (i.e., a polymer ring with one crosslink) studied by Warner [5] and present its full solution. We first confirm the results from Warner, which have been derived only for low frequencies and low Rouse mode index, but show secondly the exact solution in the entire frequency and mode domain. Moreover the technique introduced here opens new ways to study Langevin dynamics of constrained systems.

The paper is organized as follows. In section II the physical model - a generalized version of the Rouse model with internal distance constraints - is introduced. Section III summarizes some of the basic theorems regarding Langevin dynamics to be used later on. In section IV the main calculational body of the paper - the general mathematical formalism for handling internal distance constraints is developed in detail. Our treatment is a generalization of a method previously developed for computing statistical properties of randomly crosslinked Gaussian structures, i.e., ideal polymer networks [6,7]. In section $\mathrm{V}$ an application of the method to diffusional motion of a single crosslink is given (the Warner problem). Section VI contains a short discussion of main results and outlook.

\section{ROUSE MODEL WITH INTERNAL DISTANCE CONSTRAINTS}

As a minimal model for the dynamics of a Gaussian chain subject to internal distance constraints we consider a generalized version of the classical Rouse model [3]. Its discrete version is a bead-spring model, where the motion of the beads (monomers) is governed by the coupled set of Langevin equations

$$
\zeta \frac{d \mathbf{R}_{i}(t)}{d t}=-\nabla_{\mathbf{R}_{i}} \mathcal{H}_{0}\left(\left\{\mathbf{R}_{i}\right\}\right)+\mathbf{F}_{i}(t) .
$$


In this equation of motion the inertial term is omitted as usual. $\zeta$ denotes the inverse mobility or friction constant, and $\mathbf{R}_{i}(t)(i=0, \ldots, N)$ are the trajectories of the monomers in 3-dimensional space. The stochastic forces $\mathbf{F}_{i}(t)$ are assumed to be $\delta$-correlated with first and second moments given by [4]

$$
\begin{aligned}
\left\langle F_{i}^{\alpha}(t)\right\rangle & =0 \\
\left\langle F_{i}^{\alpha}(t) F_{j}^{\beta}\left(t^{\prime}\right)\right\rangle & =2 \zeta k_{\mathrm{B}} T \delta_{i j} \delta_{\alpha \beta} \delta\left(t-t^{\prime}\right) .
\end{aligned}
$$

Superscripts $\alpha, \beta=x, y, z$ represent the 3 -dimensional Cartesian coordinates. In the classical Rouse model excluded volume interaction and hydrodynamic forces are disregarded and only elastic forces between monomers are retained in the Hamiltonian. Here we consider a more general form of the Rouse model with an extra potential to allow for modelling the internal distance constraints

$$
\beta \mathcal{H}_{0}=\frac{3}{2 a^{2}} \sum_{i=1}^{N}\left(\mathbf{R}_{i}-\mathbf{R}_{i-1}\right)^{2}+\frac{3}{2 \varepsilon^{2}} \sum_{e=1}^{M}\left(\mathbf{R}_{i_{e}}-\mathbf{R}_{j_{e}}\right)^{2}
$$

The first term in the Hamiltonian represents the connectivity of a Gaussian chain with persistence length $a$, whereas the second term models the crosslinks. In particular we are concerned with permanent constraints when a monomer, say $i_{1}$, is linked to another monomer labeled by $j_{1}$. For more than one crosslink a whole set C of crosslink "coordinates" is needed to specify all junctions in the system

$$
\mathrm{C}=\left(i_{1}, j_{1}\right), \ldots,\left(i_{e}, j_{e}\right), \ldots,\left(i_{M}, j_{M}\right)
$$

For example, depending on $\mathrm{C}$ the object under investigation can be a flexible ring polymer, a two-dimensional membrane, or a rubber network (figure 1).

Although the theory will be developed for arbitrary coupling constant $\varepsilon$ two scenarios are of special relevance. For $\varepsilon \rightarrow 0$ it has been shown [6] that the Hamiltonian (3) is suitable to model hard $\delta$-constraints (the classical crosslinks) of the form

$$
\prod_{e=1}^{M} \delta\left(\mathbf{R}_{i_{e}}(t)-\mathbf{R}_{j_{e}}(t)\right)
$$


The case $\varepsilon \rightarrow \infty$ leads to the well-known problem of a free chain which serves here as a reference state. One might be worried that the above model is ill-defined and might diverge in the limit $\varepsilon \rightarrow 0$. It will be shown in section IV that the converse is true and that a surprisingly simple solution can be obtained for this special limit. As shown in the earlier paper on the static properties, ref. [6], it is important to take the limit $\varepsilon \rightarrow 0$ at the very end of the calculaton. This procedure ensures firstly, that no mathematical problems occure and secondly that in this case hard crosslink constraints is treated properly. Before going into more of the calculational details some of the basic definitions and notations regarding Langevin dynamics are summarized in the next section.

\section{LANGEVIN DYNAMICS OF IDEAL POLYMERS: PRELIMINARIES}

Consider the generalized Ornstein-Uhlenbeck process specified by equations (1)-(3). For calculational simplicity matrix notation will be used. We define $N+1$ dimensional "super-vectors" with three dimensional vector components to account for the positions of all monomers $\mathbf{R}(t)=\left(\mathbf{R}_{0}(t), \ldots, \mathbf{R}_{N}(t)\right)^{\dagger}$ and for the stochastic forces acting upon them $\mathbf{F}(t)=\left(\mathbf{F}_{0}(t), \ldots, \mathbf{F}_{N}(t)\right)^{\dagger}$. The dagger denotes the conjugate complex of the transposed vector. Furthermore, the $N+1$ dimensional connectivity (Kirchhoff) matrix is introduced as

$$
\mathcal{M}(z)=\omega_{0}\left(\mathcal{W}_{0}+\frac{1}{z} \sum_{e=1}^{M} \mathcal{X}\left(i_{e}, j_{e}\right)\right)
$$

where

$$
\mathcal{W}_{0}=\left(\begin{array}{rrrrr}
1 & -1 & 0 & \cdots & 0 \\
-1 & 2 & -1 & & \vdots \\
0 & \ddots & \ddots & \ddots & 0 \\
\vdots & & -1 & 2 & -1 \\
0 & \cdots & 0 & -1 & 1
\end{array}\right)
$$

is the Wiener matrix associated with the polymer "backbone", and 


$$
\mathcal{X}\left(i_{e}, j_{e}\right)=\left(\begin{array}{ccccccc}
0 & 0 & \cdots & 0 & \cdots & 0 & 0 \\
\vdots & \vdots & 0 & & \vdots & \vdots \\
0 & 1 & & \vdots & & -1 & 0 \\
0 & 0 & \cdots & 0 & \cdots & 0 & 0 \\
0 & -1 & & \vdots & & 1 & 0 \\
\vdots & \vdots & 0 & & \vdots & \vdots \\
0 & 0 & \cdots & 0 & \cdots & 0 & 0
\end{array}\right) \leftarrow i_{e} \text {-th row }
$$

models a single crosslink. For further use we note that a characteristic time scale is given by the inverse of the "frequency"

$$
\omega_{0}=\frac{3 k_{\mathrm{B}} T}{a^{2} \zeta} .
$$

The dimensionless parameter $z=(\varepsilon / a)^{2}$ in equation (6) is used to enforce the crosslinking constraints. With the above definitions the system of stochastic differential equations (1) cast into matrix form reads

$$
\frac{d \mathbf{R}(t)}{d t}+\mathcal{M}(z) \mathbf{R}(t)=\frac{1}{\zeta} \mathbf{F}(t) .
$$

Some of the physical quantities of interest and their interrelations are listed below. More details can be found for example in reference [8]. The Green's function to equation (10) is given by

$$
\mathcal{G}(t)=\lim _{z \rightarrow 0} e^{-\mathcal{M}(z) t} .
$$

For $z \rightarrow 0$ the case of hard $\delta$-constraints is recovered. Otherwise $z$ is an additional distance parameter in the model. Of great importance in the following derivation is the Laplace transform (resolvent) of the matrix $\mathcal{M}$

$$
\mathcal{R}(\omega) \equiv \int_{0}^{\infty} d t e^{-i \omega t} \mathcal{G}(t)=\lim _{z \rightarrow 0}(i \omega \mathcal{I}+\mathcal{M}(z))^{-1}
$$

where $\mathcal{I}$ denotes the identity matrix. 
¿From equation (2) the spectral matrix of the Langevin forces $\mathbf{F}(t)$ is found to be

$$
\left\langle\tilde{\mathbf{F}}(\omega) \tilde{\mathbf{F}}^{\dagger}\left(\omega^{\prime}\right)\right\rangle=12 \pi k_{\mathrm{B}} T \zeta \delta\left(\omega-\omega^{\prime}\right) \mathcal{I},
$$

where

$$
\tilde{\mathbf{F}}(\omega)=\int_{-\infty}^{\infty} d t e^{-i \omega t} \mathbf{F}(t)
$$

is the Fourier transform of the stochastic forces. By $\mathbf{F}_{i} \mathbf{F}_{j}$ we mean the usual 3-dimensional scalar vector product, whereas $\mathbf{F F}^{\dagger}$ is used for outer vector products. Fourier transforms are always denoted by tilde.

A formal solution to equation (10) can be obtained by Rice's method [8]. The spectral density matrix for the stochastic variable $\mathbf{R}(t)$ can be derived by use of (13) and Fourier transformation of (10)

$$
\left\langle\tilde{\mathbf{R}}(\omega) \tilde{\mathbf{R}}^{\dagger}\left(\omega^{\prime}\right)\right\rangle=12 \pi D \delta\left(\omega-\omega^{\prime}\right) \mathcal{R}(\omega) \mathcal{R}^{\dagger}\left(\omega^{\prime}\right),
$$

with the diffusion coefficient $D$ given by

$$
D=k_{\mathrm{B}} T / \zeta \text {. }
$$

Of primary interest for the diffusional behavior is the two-time correlation function matrix defined as

$$
\mathcal{C}\left(t, t^{\prime}\right)=\left\langle\left(\mathbf{R}(t)-\mathbf{R}\left(t^{\prime}\right)\right)\left(\mathbf{R}(t)-\mathbf{R}\left(t^{\prime}\right)\right)^{\dagger}\right\rangle .
$$

Finally a steady-state solution for $\mathcal{C}\left(t, t^{\prime}\right)$ in terms of the resolvent (12) is easily derived from the expression for the spectral density matrix in (15)

$$
\mathcal{C}\left(t-t^{\prime}\right)=\frac{12 D}{\pi} \int_{0}^{\infty} d \omega\left(1-\cos \omega\left(t-t^{\prime}\right)\right) \mathcal{R}(\omega) \mathcal{R}(\omega)^{\dagger} .
$$

In the following study our primary goal will be to find a general approach to calculate the resolvent $\mathcal{R}(\omega)$, equation (12), for an arbitrary set of crosslinking constraints C, equation (4). From there Green's function and correlation functions can in principle be obtained by use of the standard formulas presented in this section. Although $\mathcal{M}(z)$ is a matrix which highly depends on all the details of $\mathrm{C}$ (the crosslink positions), substantial progress can be made by invoking the following exact method. 


\section{CALCULATION OF THE RESOLVENT $\mathcal{R}(\omega)$}

The first step in deriving a general expression for $\mathcal{R}(\omega)$ for hard crosslinks is to find a way to perform the limit $z \rightarrow 0$ in equation (12). This is an interesting problem in its own right which so far could only been handled by introducing a finite cutoff at $z=1$ and successive crude variational estimates. Here we present an analytically exact approach that can overcome these difficulties. The mathematical trick is to utilize an additional symmetry of the crosslink term in (8) by writing the complete crosslink contribution in (6) in form of a dyadic (outer vector) product

$$
\sum_{e=1}^{M} \mathcal{X}\left(i_{e}, j_{e}\right)=\mathcal{U} \mathcal{U}^{\dagger}
$$

where

$$
\mathcal{U}(\mathrm{C}) \equiv\left(\mathbf{u}_{1}, \ldots, \mathbf{u}_{M}\right)
$$

has been introduced as the $(N+1) \times M$ rectangular matrix with each of its $M$ column vectors given by

$$
\mathbf{u}_{e}=\mathbf{e}_{i_{e}}-\mathbf{e}_{j_{e}} \quad, \quad(e=1, \ldots, M) .
$$

Here $\mathbf{e}_{i_{e}}$ represents the $N+1$ dimensional unit vector with 1 in the $i_{e}$ th position, and 0 otherwise. Thus $\mathcal{U}(\mathrm{C})$ has only $2 M$ elements not equal to zero that contain complete information about all crosslink positions. In the above notation each crosslink is uniquely represented by a vector $\mathbf{u}_{e}$. Note that all vectors $\mathbf{u}_{e}, e=1, \ldots, M$ are linearly independent for tetrafunctional crosslinks. Combining equations (6), (12) and (19) the resolvent cast in matrix form reads

$$
\mathcal{R}(\omega)=\frac{1}{\omega_{0}} \lim _{z \rightarrow 0}\left(i \frac{\omega}{\omega_{0}} \mathcal{I}+\mathcal{W}_{0}+\frac{1}{z} \mathcal{U} \mathcal{U}^{\dagger}\right)^{-1}
$$

It is convenient to decompose (22) into a singular and nonsingular part with the nonsingular part being 


$$
\mathcal{W}=i \frac{\omega}{\omega_{0}} \mathcal{I}+\mathcal{W}_{0}
$$

It is well-known in the mathematical literature that if the inverse of $\mathcal{W}$ exists, then the inverse in equation (22) is given by

$$
\begin{aligned}
(\mathcal{W} & \left.+\frac{1}{z} \mathcal{U U}^{\dagger}\right)^{-1}=\mathcal{W}^{-1} \\
& \times\left(\mathcal{I}-\mathcal{U}\left(z \mathcal{I}+\mathcal{U}^{\dagger} \mathcal{W}^{-1} \mathcal{U}\right)^{-1} \mathcal{U}^{\dagger} \mathcal{W}^{-1}\right)
\end{aligned}
$$

This theorem can be directly verified by matrix multiplication. The latter identity is also known as Sherman-Morrison formula [9].

\section{A. The limit $z \rightarrow 0$}

There are two subtle points about the existence of the right hand side of (24). First we require $\mathcal{W}^{-1}$ to exist. The only critical case arises if $\omega=0$, i.e., when $\mathcal{W}=\mathcal{W}_{0}$ in (23). The problem here is that $\mathcal{W}_{0}$ is only positive semi definite and there is one mode with eigenvalue 0 from translational invariance. This can be directly seen from the definition of $\mathcal{W}_{0}$ in $(7)$ which is a row (column) constant matrix. However, even in the semi definite case the above theorem remains valid if $\mathcal{W}^{-1}$ denotes a generalized inverse of $\mathcal{W}$ as was proved in reference [10].

Secondly from the definition of $\mathbf{u}_{e}$ in (21) it is easily verified that for tetrafunctional crosslinks all $M$ vectors $\mathbf{u}_{e}$ are linearly independent. Thus in general the kernel $z \mathcal{I}+\mathcal{U}^{\dagger} \mathcal{W}^{-1} \mathcal{U}$ will be a positive definite matrix of dimension $M$ and full rank which has only positive eigenvalues for all nonnegative values of $z$. As a consequence performing the $z \rightarrow 0$ limit in equation (24) leads to a well-defined expression for the resolvent

$$
\mathcal{R}(\omega)=\frac{1}{\omega_{0}} \mathcal{W}^{-1}\left(\mathcal{I}-\mathcal{U}\left(\mathcal{U}^{\dagger} \mathcal{W}^{-1} \mathcal{U}\right)^{-1} \mathcal{U}^{\dagger} \mathcal{W}^{-1}\right)
$$

The first term is the linear chain (Rouse) model, whereas the second part arises entirely from the effect of crosslinking. Although the case of general crosslinking potential $z$ is still implicit in the basic formula (24), we will restrict ourselves in the following discussion to 
the somewhat simpler case $z=0$, i.e., hard $\delta$-constraints. Equations (24) and (25) are formally exact solutions to the problem posed in equations (1)-(4). The further evaluation of $\mathcal{R}(\omega)$ for specific realizations of crosslinks $\mathrm{C}$ can be split into two parts and is discussed in subsequent sections.

\section{B. Resolvent of $\mathcal{W}_{0}$}

Evaluation of the inverse of $\mathcal{W}$ in $(25)$ can in principle be done by full diagonalization of $\mathcal{W}_{0}$ which is tridiagonal. For calculational simplicity we consider here only the cyclic counterpart of $\mathcal{W}_{0}$ with periodic boundary conditions

$$
\mathcal{W}_{0}=\left(\begin{array}{rrrrr}
2 & -1 & 0 & \cdots & -1 \\
-1 & 2 & -1 & \cdots & 0 \\
\vdots & \ddots & \ddots & \ddots & \vdots \\
0 & \cdots & -1 & 2 & -1 \\
-1 & \cdots & 0 & -1 & 2
\end{array}\right) .
$$

Both models (7) and (26) are known to obey the same Rouse dynamics in the limit $N \rightarrow \infty$

[11]. Physically the latter situation represents a flexible ring polymer. The eigensystem to (26) is of particular simple form since it is a circulant. The eigenvalues read

$$
\lambda_{k}=4 \sin ^{2} \frac{\pi k}{N+1} \quad, \quad k=0, \ldots, N
$$

The modal matrix of $(26)$ is the Fourier matrix $\mathcal{F}$ [14 with matrix elements

$$
[\mathcal{F}]_{k l}=\frac{1}{\sqrt{N+1}} \exp \frac{2 \pi i k l}{N+1}, k, l=0, \ldots, N
$$

Spectral decomposition leads to the well-known representation of the inverse $\mathcal{W}^{-1}$ in terms of its eigenvalues

$$
\left[\mathcal{W}^{-1}\right]_{k l}=\frac{1}{N+1} \sum_{n=0}^{N} \frac{\exp \frac{2 \pi i n(k-l)}{N+1}}{i \omega / \omega_{0}+\lambda_{n}}
$$




\section{Discussion of kernel}

The remaining calculational task for determining $\mathcal{R}(\omega)$ is the evaluation of the kernel function in the second part of (25)

$$
\mathcal{K}(\omega ; \mathrm{C}) \equiv\left(\mathcal{U}^{\dagger} \mathcal{W}^{-1} \mathcal{U}\right)^{-1}
$$

Since $\mathcal{K}(\omega ; \mathrm{C})$ depends on all the crosslink positions $\mathrm{C}=\left(i_{1}, j_{1}\right), \ldots,\left(i_{M}, j_{M}\right)$ via $\mathcal{U}$ no further analytical progress is possible without specifying the crosslink in the system. On the other hand, from the mathematical structure of $\mathcal{K}(\omega ; \mathrm{C})$ most problems of interest fall into one of the following three categories. Only one of these will be considered in detail in section $\mathrm{V}$.

(i) The number of crosslinks $M$ is small. Since $\mathcal{K}(\omega ; \mathrm{C})$ requires inversion of an $M \times M$ matrix analytical progress is always possible if $M$ is not too large. A particularly simple problem is treated in the next section when we consider the dynamics of a polymer shaped like the figure-of-eight (figure 1a).

(ii) Another special case arises when $M$ is large, but there is some additional pattern in the structure of $\mathcal{U}$. Examples of this kind are illustrated in figures $1 \mathrm{~b}$ and 1c. In particular the sketch in $1 \mathrm{~b}$ shows an example of a macromolecule with distance constraints $z \neq 0$, i.e., the more general case governed by equation (24). For the above examples $\mathcal{K}$ can be calculated as a consequence of the regularity of the crosslink positions. We will report on these systems in a separate publication.

(iii) The third important category arises when $M$ is large and the crosslink positions $\mathrm{C}$ in (4) are picked at random. This is the case of a polymer gel (figure 1d). Here one has to resort to numerical computation of $\mathcal{K}(\omega ; \mathrm{C})[7]$. However, there is still a huge calculational advantage with (25). For a polymer network we have in general $M \ll N$. Equation (25) requires "only" the inverse of an $M \times M$ matrix [7] and not of the complete $N \times N$ connectivity matrix as is commonly believed in the polymer literature [12,13. An analytic approach to the network problem would be to perform the quenched average of the resolvent over the crosslink positions C. The latter problem is a key problem in current network research and 
has not been analytically solved even for the static problem.

Before calculating $\mathcal{R}(\omega)$ for a specific example, we want to establish some remarkable and general properties of the operators in equation (25). Consider the crosslink part in (25)

$$
\mathcal{V} \equiv \mathcal{W}^{-1} \mathcal{U}\left(\mathcal{U}^{\dagger} \mathcal{W}^{-1} \mathcal{U}\right)^{-1} \mathcal{U}^{\dagger} \mathcal{W}^{-1}
$$

By elementary matrix multiplication it is found that

$$
\mathcal{V W} \mathcal{V}=\mathcal{V}, \mathcal{U}^{\dagger} \mathcal{V}=\mathcal{U}^{\dagger} \mathcal{W}^{-1}, \mathcal{V U}=\mathcal{W}^{-1} \mathcal{U}
$$

A matrix with these properties is said to be a generalized projector to $\mathcal{W}^{-1}$. Furthermore

$$
(\mathcal{W V})^{2}=\mathcal{W} \mathcal{V},(\mathcal{V W})^{2}=\mathcal{V W}
$$

are idempotents whose eigenvalues are known exactly: $\lambda_{1}=1$ and $\lambda_{2}=0$ with degeneracies $M$ and $N-M$. By use of the above results it is easy to prove that the resolvent satisfies a remarkable orthogonality relation

$$
\mathcal{U}^{\dagger} \mathcal{R}(\omega)=\mathcal{R}(\omega) \mathcal{U}=0
$$

Equation (34) is valid for arbitrary crosslink positions $\mathrm{C}$ and independent of the specific crosslink topology of the system under investigation.

\section{DIFFUSIONAL MOTION OF A SINGLE CROSSLINK}

As the simplest possible application of the method developed in section IV we consider the figure-of-eight shaped polymer depicted in figure 1a. What we have in mind is to model the dynamics of a single crosslink in an ideal dilute network when the distance between crosslinks is large [5]. It is expected that monomers in the neighborhood of the crosslink are somewhat affected by the slower dynamics of the crosslink [1,5, 15].

A suitable realization of the system in figure 1a would be 


$$
\mathcal{U}(\mathrm{C})=\mathbf{u}_{1}=\mathbf{e}_{0}-\mathbf{e}_{\frac{N+1}{2}}
$$

That is, monomer 0 is linked to monomer $(N+1) / 2$. The main calculational task is to determine the kernel function, equation (30), of the system. From (35) and with $\mathcal{W}^{-1}$ given by (29) we get immediately

$$
\mathcal{K}(\omega)=\left(\frac{4}{N+1} \sum_{n o d d} \frac{1}{i \omega / \omega_{0}+\lambda_{n}}\right)^{-1},
$$

where the summation includes only the odd terms. For the diffusional motion the quantity of interest is the self-correlation function contained in the diagonal elements of the correlation matrix (18)

$$
\left[\mathcal{C}\left(t-t^{\prime}\right)\right]_{s s}=\left\langle\left(\mathbf{R}_{s}(t)-\mathbf{R}_{s}\left(t^{\prime}\right)\right)^{2}\right\rangle
$$

where $s$ is the distance of the $s$ th monomer with respect to the crosslink at position $s=0$. Typical terms and manipulations in the straight-forward derivation which is not carried out in detail are of the form

$$
\begin{aligned}
& \frac{1}{N+1} \sum_{n \text { odd }} \frac{\exp \frac{2 \pi i s n}{N+1}}{i \omega / \omega_{0}+\lambda_{n}} \\
\simeq & \frac{1}{2} \int_{0}^{1} d x \frac{\exp (2 \pi i s x)}{i \omega / \omega_{0}+4 \sin ^{2}(\pi x)} \\
\simeq & \frac{1}{4} \sqrt{\frac{\omega_{0}}{\omega}} \exp \left(-\frac{i \pi}{4}-(1+i)|s| \sqrt{\frac{\omega}{2 \omega_{0}}}\right) .
\end{aligned}
$$

In deriving the first integral we have performed the $N \rightarrow \infty$ limit. The latter expression was obtained by setting $\sin (\pi x) \simeq \pi x$. Only the final result for the self-correlation function (37) is quoted here

$$
\left\langle\left(\mathbf{R}_{s}(t)-\mathbf{R}_{s}\left(t^{\prime}\right)\right)^{2}\right\rangle=A\left(s,\left|t-t^{\prime}\right|\right) 2 a^{2} \sqrt{\frac{\omega_{0}\left|t-t^{\prime}\right|}{\pi}} .
$$

The time-dependent prefactor is given by

$$
\begin{aligned}
A\left(s,\left|t-t^{\prime}\right|\right) & =1-\frac{1}{2 \sqrt{2 \pi}} \int_{0}^{\infty} d x \frac{1-\cos x}{x^{3 / 2}} \\
& \times e^{-s^{\prime} \sqrt{x}}\left(\cos \left(s^{\prime} \sqrt{x}\right)+\sin \left(s^{\prime} \sqrt{x}\right)\right) \\
& =1-\frac{1}{4 \pi} \int_{0}^{\infty} d y \cos \left(\frac{y s^{\prime 2}}{2}-\frac{\pi}{4}\right) \frac{\log \left(1+y^{2}\right)}{y^{3 / 2}}
\end{aligned}
$$


which scales with

$$
s^{\prime} \equiv \sqrt{\frac{2 s^{2}}{\omega_{0}\left|t-t^{\prime}\right|}} .
$$

The complicated integral in (41) is plotted in figure 2. The asymptotic behavior for small values of $s^{\prime}$ is governed by the expansion

$$
A\left(s^{\prime}\right)=\frac{1}{2}+\frac{s^{\prime} \sqrt{\pi}}{2 \sqrt{2}}-\frac{s^{\prime 2}}{4}+\mathcal{O}\left(s^{\prime 3}\right) .
$$

In particular for the diffusional motion of the crosslink $(s=0)$, we find $A=1 / 2$ which is exactly half the diffusion constant of an unconstrained monomer in the Rouse model [16]. The finding is in agreement with the result in reference [5] based on the method of Lagrangian multipliers. For $s \rightarrow \infty$ (monomers that are sufficiently far from the crosslink) we recover the diffusion law of the classical Rouse model $(A=1)$ which was first derived by de Gennes [16].

In addition we obtain the crossover from the slower dynamics of the crosslink to that of a "free" monomer in the classical Rouse model as $s$ is varied from zero to infinity. The crossover takes place on time scales of the order

$$
\tau_{s}=s^{2} / \omega_{0}=(s a)^{2} /(3 D)
$$

where sa measures the distance of the monomer from the crosslink (figure 3). Interestingly a monomer begins to feel the presence of the crosslink only after a timespan of the order $\tau_{s}$.

The two limiting cases $A=1 / 2$ relevant for the slower dynamics of the crosslink and $A=1$ for the "free" chain segments far away from the crosslink are expected on physical grounds (dashed lines in figure 3). An "inner" chain segment has only two neighbors, whereas the crosslink is surrounded by four neighbors. Thus in general for a monomer with functionality $f$ a prefactor $A(f)=f / 2$ is expected as was pointed out previously [5].

\section{CONCLUSION}

Within the framework of the Rouse model we have proofed that an exact solution for the Langevin dynamics of a polymer subject to hard delta constraints exists when excluded 
volume and hydrodynamic forces are neglected. The fundamental and general result for the resolvent, equation (25), was derived for an arbitrary crosslink configuration making our results also applicable to the challenging problem of a random network.

In this investigation we restricted ourselves to the simplest physical scenario, where only Rouseian dynamics was involved. This case was deliberately chosen to highlight the principal mathematical difficulties. As a special application we studied the dynamics of the figure-ofeight shaped polymer depicted in figure 1a. In contrast to an earlier attempt by Warner [5] based on Lagrangian multipliers which yielded only two limiting cases $s \rightarrow 0$ and $\infty$, the full solution could be derived by our method. Moreover, our result allows for computation of the dynamic scattering function [16] and comparison with experimental data taken in the dry network state. A detailled comparison will be studied in a future, less formal paper.

For the physically more realistic scenario of a swollen network in a Theta solvent further generalizations are required like taking hydrodynamic interaction into account. A generalized version of the equation of motion (1) would read

$$
\zeta \frac{d \mathbf{R}_{i}(t)}{d t}=\sum_{j} \boldsymbol{\Theta}_{i, j}\left(-\nabla_{\mathbf{R}_{j}} \mathcal{H}_{0}\left(\left\{\mathbf{R}_{j}\right\}\right)+\mathbf{F}_{j}(t)\right)
$$

where $\boldsymbol{\Theta}_{i, j}$ is the Oseen tensor. Although the above equation becomes analytically untractable, for most experimental situations a pre-averaged treatment is well justified [4]. This computation is left for future work.

\section{ACKNOWLEDGMENTS}

MPS wishes to acknowledge financial support from the Deutsche Forschungsgemeinschaft, Sonderforschungsbereich 262. 


\section{REFERENCES}

[1] S. F. Edwards, J. Phys. A: Math. Gen. 7, 318 (1974).

[2] S. F. Edwards in Polymer Networks, eds. A. J. Chompff and S. Newman (Plenum Press, New York, 1971); R. T. Deam and S. F. Edwards, Proc. Trans. R. Soc. London A 280 (1976) 317; R. C. Ball and S. F. Edwards, Macromolecules 13 (1980) 748.

[3] P. E. Rouse, J. Chem. Phys. 21, 1272 (1953).

[4] M. Doi and S. F. Edwards, The Theory of Polymer Dynamics (Clarendon Press, Oxford, 1986), chap. 4.

[5] M. Warner, J. Phys. C: Solid State Phys. 14, 4985 (1981).

[6] M. P. Solf and T. A. Vilgis, J. Phys. A: Math. Gen. 28, 6655 (1995).

[7] M. P. Solf and T. A. Vilgis, J. Phys. France I, submitted.

[8] H. Risken, The Fokker-Planck Equation (Springer-Verlag, Berlin, 1989), chap. 3.

[9] P. Lancaster and M. Tismenetsky, Theory of Matrices (Academic Press, San Diego, 1985).

[10] T. O. Lewis and T. G. Newman, SIAM J. Appl. Math. 16, 701 (1968).

[11] B. H. Zimm and R. W. Kilb, J. Polymer Sci. 37, 19 (1959).

[12] B.E. Eichinger, J.E. Martin, J. Chem. Phys. 69, 4595 (1978)

[13] M. Schulz, P. Reineker, M. Möller, J. Chem. Phys. 103, 10701 (1995)

[14] P. J. Davis, Circulant Matrices (Wiley, New York, 1979).

[15] T. A. Vilgis and F. Boué, J. Polymer Sci.: Part B 26, 2291 (1988).

[16] P. G. de Gennes, Physics 3, 37 (1967). 


\section{FIGURES}

FIG. 1. Examples of different crosslinking topologies. (a) The polymer shape discussed in section V. $a$ is the persistence length of the polymer backbone. The hard crosslink constraint is enforced by $\varepsilon \rightarrow 0$. (b) A ladder shaped polymer with $\varepsilon \neq 0$. (c) Two-dimensional membrane. (d) Random network.

FIG. 2. Prefactor $A\left(s^{\prime}\right)$, equation (41). The dashed lines represent the asymptotic behavior for small and large values of $s^{\prime}$.

FIG. 3. Crossover of the mean squared displacement from the dynamics of a "free" Rouseian monomer (upper dashed line) to the slower dynamics of a crosslink (lower dashed line). 